\title{
Effect of higher implant density on curve correction in dystrophic thoracic scoliosis secondary to neurofibromatosis Type 1
}

\author{
Yang Li, PhD, Xinxin Yuan, PhD, Shifu Sha, PhD, Zhen Liu, PhD, Weiguo Zhu, PhD, Yong Qiu, MD, \\ Bin Wang, MD, Yang Yu, MD, and Zezhang Zhu, MD \\ Department of Spine Surgery, Drum Tower Hospital, Nanjing University Medical School, Nanjing, China
}

\begin{abstract}
OBJECTIVE The aim of this study was to investigate how implant density affects radiographic results and clinical outcomes in patients with dystrophic scoliosis secondary to neurofibromatosis Type 1 (NF1).

METHODS A total of 41 patients with dystrophic scoliosis secondary to NF1 who underwent 1-stage posterior correction between June 2011 and December 2013 were included. General information about patients was recorded, as were preoperative and postoperative scores from Scoliosis Research Society (SRS)-22 questionnaires. Pearson correlation analysis was used to analyze the associations among implant density, coronal Cobb angle correction rate and correction loss at last follow-up, change of sagittal curve, and apical vertebral translation. Patients were then divided into 2 groups: those with low-density and those with high-density implants. Independent-sample t-tests were used to compare demographic data, radiographic findings, and clinical outcomes before surgery and at last follow-up between the groups.
\end{abstract}

RESULTS Significant correlations were found between the implant density and the coronal correction rate of the main curve $(r=0.505, p<0.01)$ and the coronal correction loss at final follow-up $(r=-0.379, p=0.015)$. There was no significant correlation between implant density and change of sagittal profile $(p=0.662)$ or apical vertebral translation $(p$ $=0.062)$. The SRS-22 scores improved in the appearance, activity, and mental health domains within both groups, but there was no difference between the groups in any of the SRS-22 domains at final follow-up ( $p>0.05$ for all).

CONCLUSIONS Although no significant differences between the high- and low-density groups were found in any of the SRS-22 domains at final follow-up, higher implant density was correlated with superior coronal correction and less postoperative correction loss in patients with dystrophic NF1-associated scoliosis.

https://thejns.org/doi/abs/10.3171/2017.4.PEDS171

KEY WORDS neurofibromatosis Type 1; implant density; radiographic results; clinical outcomes; correction rate; correction loss; spine

$\mathrm{N}$ EUROFIBROMATOSIS Type 1 (NF1) is a complex autosomal-dominant hereditary disorder that commonly leads to the development of scoliosis. Two distinct forms of scoliotic curves have been documented to occur in NF1 based on the absence or presence of skeletal dysplasia on plain radiographic evaluation: a nondystrophic curve which resembles that seen in idiopathic scoliosis, and a dystrophic curve which is highly progressive due to dystrophic changes such as vertebral wedging, vertebral rotation, and rib penciling. ${ }^{3,7,8}$ Although pedicle screwbased spinal fusion has been widely used for surgical correction of such NF1-asscoiated scoliosis, placement of pedicle screws is challenging in dystrophic cases because its anchorage points for internal fixation are poor due to vertebral destruction, leading to lower implant density. ${ }^{12,15}$

Recent advances in intraoperative assistive techniques (e.g., O-arm navigation) provide a safe process to insert more pedicle screws, leading to higher implant density in scoliosis surgery. ${ }^{12}$ However, whether the increased implant density would result in better curve correction needs to be further explored. Over the past few decades, the relationship between the density of implant sites and surgical outcomes has been extensively investigated in idiopathic scoliosis. Limited data, however, are available in

ABBREVIATIONS AVT = apical vertebral translation; $\mathrm{HD}=$ high density; LD = low density; MCID = minimal clinically important difference; NF1 = neurofibromatosis Type 1; SRS = Scoliosis Research Society. 
this unique population of patients with dystrophic scoliosis secondary to NF1.

There are biomechanical and anatomical differences between idiopathic scoliosis and dystrophic NF1-associated scoliosis; the latter has a tendency to progress because of its dystrophic changes, and it is usually associated with decreased bone mineral density. ${ }^{11,13,14}$ Thus, this study aimed to evaluate the relationship between implant density and clinical outcomes as well as radiographic results in patients with dystrophic NF1-associated scoliosis treated with posterior spinal fusion.

\section{Methods \\ Patient Selection}

With approval from the institutional review board, a retrospective study was conducted in 68 patients $(27$ of whom were subsequently excluded) with dystrophic NF1associated scoliosis surgically treated at our spine center between June 2011 and December 2013. A diagnosis of NF1 was made according to the criteria defined by the Consensus Development Conference of the National Institutes of Health on NF1. Radiographs, CT scans, and MR images were analyzed for evidence of dystrophic features, and patients with 3 or more of the following features were defined as having a dystrophic curve: 1) rib penciling; 2) vertebral rotation of Grade 3 or higher as measured by using the Nash and Moe method; ${ }^{17}$ 3) vertebral scalloping; 4) vertebral wedging in either the sagittal or coronal plane; 5) spindling of the transverse processes; 6) focal, short-segmented curve involving 6 or fewer vertebrae; 7) dural ectasia seen on MR image; and 8) paraspinal tumors or plexiform neurofibromas located close to the scoliotic curve on MR image. ${ }^{16}$

Patients selected for further analyses met the following criteria: 1) presented with a thoracic curve between $50^{\circ}$ and $100^{\circ} ; 2$ ) had been treated with 1-stage posterior spinal fusion and a minimum of $80 \%$ of the implants used were pedicle screws; and 3) had a minimum 2-year clinical and radiographic follow-up. Patients who had received surgery or 3-column osteotomy were excluded.

\section{Surgical Procedures}

All patients were treated with posterior pedicle screwbased instrumentation or hybrid instrumentation with hooks. If the pedicles were large enough, we preferred to select the pedicle screw fixation to ensure sufficient fixing strength. Hooks were used selectively when placement of the pedicle screw may have failed for vertebrae with severe slender or even absent pedicles. Pedicle screws were inserted using a free-hand or $\mathrm{O}$-arm navigation technique.

A contoured rod was placed first on the concave side in all patients, followed by the rod on the convex side. Correction of the deformity was performed by a combination of rod de-rotation and sequential in situ translational reduction of the apical vertebral screws toward the concave rod, with or without in situ bending of the rod. Additional correction maneuvers, including appropriate compression and/or distraction, were applied to provide 3D correction of the deformity.

The posterior elements were decorticated, and bone grafts were placed on the decorticated bed using autogenous local bone grafts in combination with autogenous iliac bone grafts. Both somatosensory and motor evoked potentials were monitored throughout the operation, and wake-up tests were routinely performed before closure.

\section{Clinical and Radiographic Evaluation}

The number of levels fused, number of implants (screws and hooks) in the construct, and implant density (the number of implants per level instrumented) were recorded. The financial data (including length of stay in the hospital, hospital charges, and implant charges) were also recorded. Thoracic Cobb angle, apical vertebral translation (AVT), and T5-12 kyphosis were measured on preoperative, early postoperative, and minimum 2-year postoperative longcassette standing radiographs. The radiographic measurements were done by 2 independent spinal surgeons, and the mean values were used for the analysis. The Scoliosis Research Society (SRS)-22 questionnaire was taken by patients before surgery and at the last follow-up; these scores served as clinical outcomes, which included subscores of 5 domains (pain, appearance, mental health, activity, and satisfaction) and a total score.

\section{Statistical Analysis}

Data were analyzed using SPSS statistical software (version 19.0; IBM SPSS Statistics) and expressed as the mean \pm SD. The Pearson coefficients were used for correlation analysis. Bivariate analysis was conducted between implant density and the radiographic parameters. Patients were then divided into 2 groups: the low-density (LD) group (defined by implant density lower than the mean number of implants per level for the entire cohort) and the high-density (HD) group (defined by implant density higher than the mean number of implants per level). Independent sample t-tests were used to compare demographic data as well as radiographic and clinical outcomes at baseline and follow-up between the groups. A p $<0.05$ was considered statistically significant.

\section{Results}

Forty-one patients ( 25 males and 16 females) with an average age of $13.0 \pm 4.6$ years (range $8-18$ years) were included in this study. The mean follow-up was $28.8 \pm 4.3$ months (range 24-39 months). The average main thoracic Cobb angle improved from $70.2^{\circ} \pm 10.1^{\circ}$ (range $51^{\circ}-98^{\circ}$ ) preoperatively to $35.6^{\circ} \pm 14.2^{\circ}$ (range $10^{\circ}-65^{\circ}$ ) postoperatively, with an average correction rate of $50 \% \pm 16 \%$. The mean correction loss at final follow-up was $5.3^{\circ} \pm 2.9^{\circ}$. The average T5-12 kyphosis decreased from $49.1^{\circ} \pm 22.3^{\circ}$ (range $18^{\circ}-72^{\circ}$ ) before surgery to $29.1^{\circ} \pm 12.1^{\circ}$ (range $12^{\circ}-66^{\circ}$ ) after surgery. The mean number of levels fused was $11.2 \pm 1.7$, with a mean implant density of 1.35 . Seven patients were operated with hybrids of pedicle screws with hooks, of whom 1 patient was operated with 3 hooks, 3 patients with 2 hooks, and 3 patients with 1 hook. The $\mathrm{O}$-arm navigation system was used in 17 patients with a mean implant density of $1.58 \pm 0.09$, which was higher than in those operated without use of the O-arm $(1.19 \pm 0.11 ; p=$ 0.000). At final follow-up, SRS-22 scores improved from 
TABLE 1. Correlation analysis of change variable and implant density

\begin{tabular}{lcc}
\hline \multicolumn{1}{c}{ Variable } & $\begin{array}{c}\text { Correlation } \\
\text { Coefficient }(r)\end{array}$ & $\begin{array}{c}p \\
\text { Value }\end{array}$ \\
\hline Correction rate of the main thoracic curve & 0.505 & $0.00^{*}$ \\
\hline Coronal correction loss & -0.379 & $0.015 \dagger$ \\
\hline Change of T5-12 kyphosis & -0.070 & 0.662 \\
\hline Change of AVT & 0.297 & 0.062 \\
\hline
\end{tabular}

* Statistically significant at $\alpha=0.01$ level.

$\dagger$ Statistically significant at $\alpha=0.05$ level.
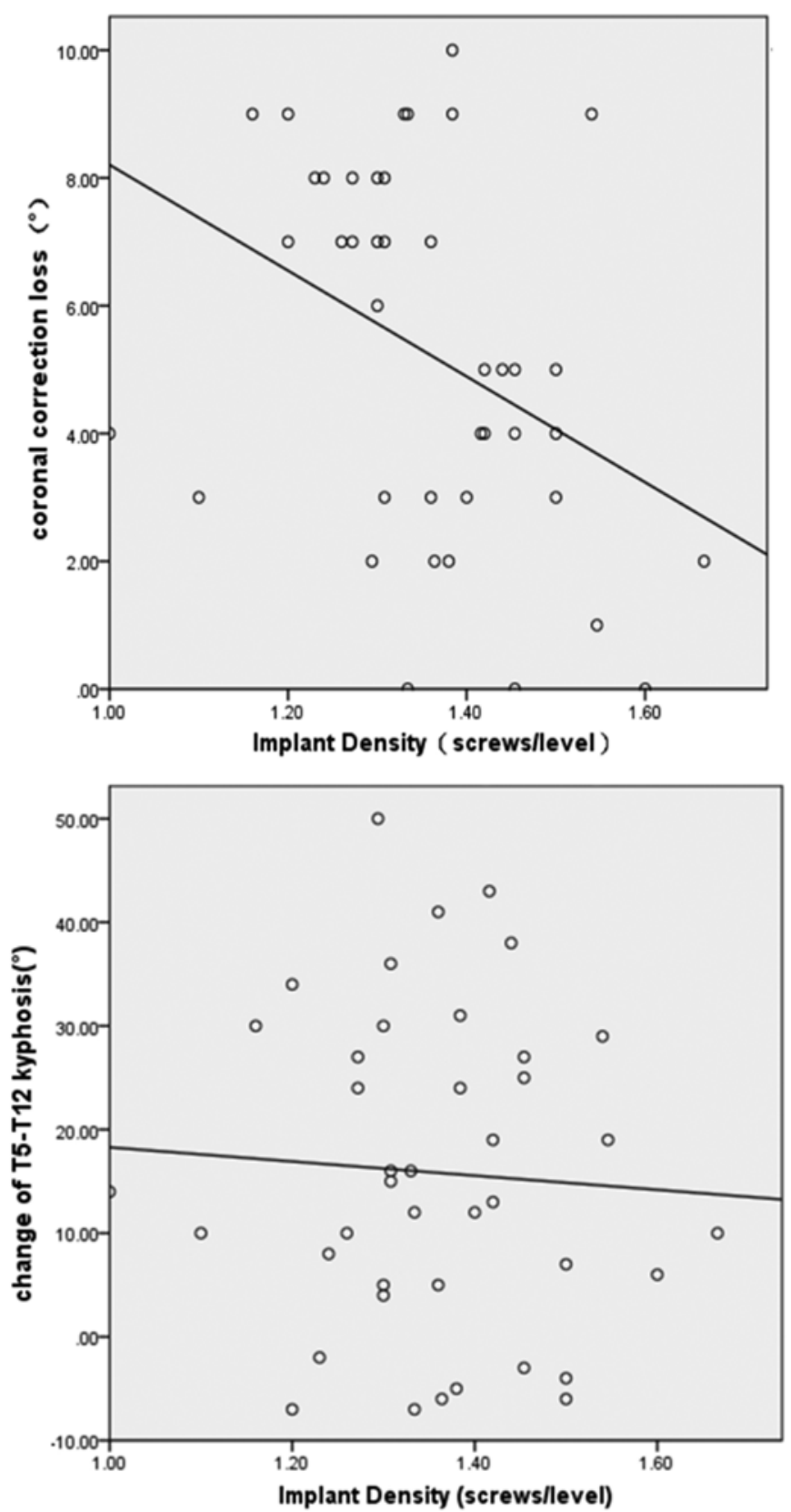

the baseline in the appearance domain $(3.3 \pm 0.21$ vs 4.2 $\pm 0.16 ; \mathrm{p}<0.05)$, activity domain $(4.1 \pm 0.19$ vs $4.3 \pm 0.22$; $\mathrm{p}<0.05)$, and mental health domain $(3.7 \pm 0.20$ vs $4.3 \pm$ $0.3 ; \mathrm{p}<0.05)$.

By bivariate analysis, implant density was found to be significantly correlated with coronal correction of the main thoracic curve $(r=0.505, p<0.01)$ and correction loss at final follow-up $(r=-0.379, p=0.015)$. However, there was no correlation between implant density and the change in T5-12 kyphosis $(\mathrm{p}=0.662)$ or correction of $\operatorname{AVT}(p=0.062)($ Table 1, Fig. 1).
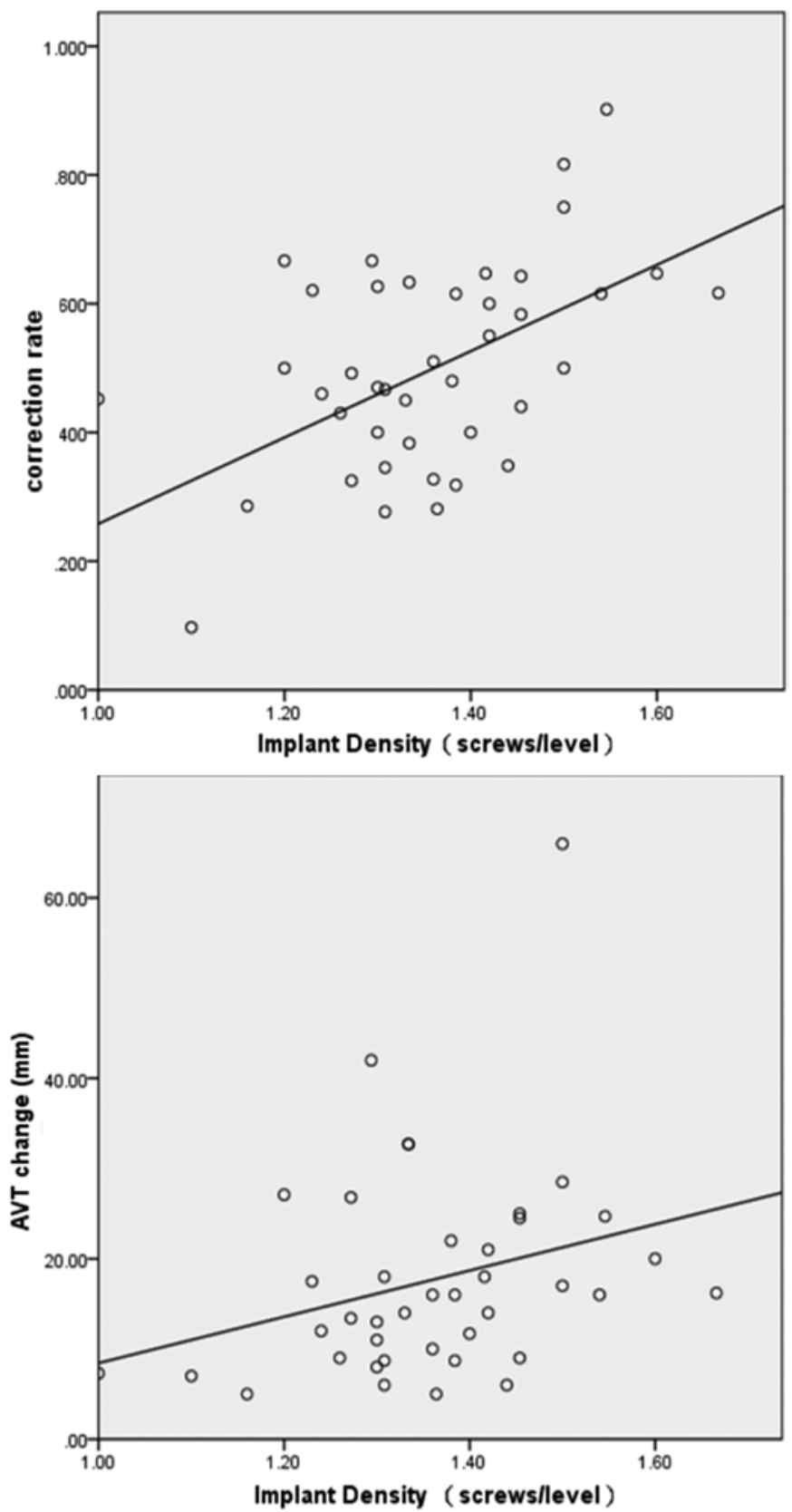

FIG. 1. Scatterplots display bivariate analysis results of implant density versus radiographic outcomes. There were significant correlations between implant density and coronal correction of the main scoliosis curve $(r=0.505, p<0.01)$ and coronal correction loss at last follow-up $(r=-0.379, p=0.015)$. No significant correlations were observed between implant density and change of sagittal curve and change of AVT. 
TABLE 2. Preoperative demographic characteristics and clinical features of patients in the LD and HD groups

\begin{tabular}{lccc}
\hline \multicolumn{1}{c}{ Variable } & LD Group & HD Group & $\mathrm{p} \mathrm{Value}$ \\
\hline No. of patients & 20 & 21 & $\mathrm{NA}$ \\
\hline Sex/no. & $\mathrm{M} / 13, \mathrm{~F} / 7$ & $\mathrm{M} / 12, \mathrm{~F} / 9$ & $\mathrm{NA}$ \\
\hline Flexibility & $24.7 \% \pm 7.5 \%$ & $22.7 \% \pm 8.1 \%$ & 0.363 \\
\hline Implant density & $1.24 \pm 0.09$ & $1.46 \pm 0.08$ & $0.000^{*}$ \\
\hline Age at op, yrs & $12.7 \pm 4.1$ & $14.6 \pm 0.08$ & 0.740 \\
\hline Follow-up, mos $^{*}$ & $29.5 \pm 4.1$ & $28.1 \pm 4.4$ & 0.585 \\
\hline Main scoliosis angle, ${ }^{\circ}$ & $72.1 \pm 12.5$ & $69.5 \pm 13.3$ & 0.420 \\
\hline AVT, mm $^{\text {Thoracic kyphosis angle, }}{ }^{\circ} \dagger$ & $43.8 \pm 10.7$ & $39.1 \pm 13.3$ & 0.306 \\
\hline
\end{tabular}

$\mathrm{NA}=$ not applicable.

Values are expressed as the mean \pm SD unless otherwise noted.

* Statistically significant at $\alpha=0.01$ level.

† T5-12 kyphosis.

The mean implant density was 1.35 . The cohort was then divided into the HD group (implant density $>1.35$ ) and the LD group (implant density $\leq 1.35$ ) for further analysis. There were 20 patients in the LD group and 21 patients in the HD group. Both groups were similar at baseline with respect to age, sex, primary curve magnitude, curve flexibility, and thoracic kyphosis (Table 2). There was no significant difference between the groups with respect to operative time, estimated blood loss, blood transfusion, or length of stay in the hospital (Table 3). Total hospital charges $(\$ 16,310.30 \pm \$ 1462.40$ vs $\$ 14,581.60$ $\pm \$ 1821.80 ; \mathrm{p}=0.002)$ and implant charges $(\$ 11,040.60 \pm$ $\$ 1191.30$ vs $\$ 9194.50 \pm \$ 1018.20 ; p=0.000)$ were significantly higher in the HD group than in the LD group.

However, patients in the HD group achieved better correction rates $(45.2 \% \pm 14.7 \%$ vs $55.2 \% \pm 16.4 \% ; \mathrm{p}<0.05)$ and less correction loss $\left(6.5^{\circ} \pm 2.6^{\circ}\right.$ vs $4.1^{\circ} \pm 2.8^{\circ} ; \mathrm{p}<$ $0.05)$ at last follow-up, despite the similar number of levels fused in both groups (Figs. 2 and 3). A total of 11 patients $(55 \%)$ in the LD group and 4 patients (19\%) in the HD group had a correction loss $>5^{\circ}$ at last follow-up. Moreover, 2 patients $(10 \%)$ in the LD group and 1 patient $(4.8 \%)$ in the HD group had a correction loss $>10^{\circ}$. With regard to the correction of thoracic kyphosis or AVT, no statistical difference was found between the groups (Table 3).

The SRS-22 scores of the appearance, activity, and mental health domains improved in both groups at final follow-up compared with baseline. However, no significant difference between the HD and LD groups was found in any of the SRS-22 domains at last follow-up, although the differences in the appearance domain nearly reached statistical significance $(\mathrm{p}=0.055)$ (Table 4).

After surgery, no CSF leakage or wound infection occurred in either group. No screw-related neurological or vascular complications were noted in either group. In addition, there were no incidences of rod fracture, screw loosening or pullout, or reoperation during follow-up.

\section{Discussion}

Although lower implant density has been recognized to be associated with higher risk of instrumentation failure, 2,6,9,19 whether higher implant density may independently predict better curve correction and improved clinical outcomes remains to be determined. In a multicenter study with 2-year follow-up, Clements et al. found the major curve correction to be positively correlated with implant density in patients with idiopathic scoliosis. ${ }^{6}$ In contrast, Quan and Gibson, as well as other scholars, observed no difference between consecutive and interval pedicle screw placement with respect to postoperative correction in the sagittal and coronal planes. ${ }^{2,9,19}$

TABLE 3. Postoperative demographic characteristics and clinical features of patients in the LD and HD groups

\begin{tabular}{|c|c|c|c|}
\hline Variable & LD Group & HD Group & $\mathrm{p}$ Value \\
\hline No. of levels fused & $11.3 \pm 2.0$ & $11.0 \pm 1.3$ & 0.640 \\
\hline No. of implants & $13.7 \pm 2.3$ & $16.3 \pm 1.3$ & $0.034^{*}$ \\
\hline Implant density & $1.24 \pm 0.09$ & $1.46 \pm 0.08$ & $0.000 \dagger$ \\
\hline Main scoliosis angle, ${ }^{\circ}$ & $39.6 \pm 14.8$ & $31.8 \pm 16.1$ & 0.123 \\
\hline Scoliosis correction rate, $\%$ & $45.2 \pm 14.7$ & $55.2 \pm 16.4$ & $0.048^{*}$ \\
\hline Coronal correction loss, $^{\circ}$ & $6.5 \pm 2.6$ & $4.1 \pm 2.8$ & $0.009 \dagger$ \\
\hline AVT, mm & $27.6 \pm 18.9$ & $18.7 \pm 12.5$ & 0.150 \\
\hline AVT correction, mm & $16.4 \pm 10.8$ & $18.8 \pm 12.6$ & 0.516 \\
\hline Thoracic kyphosis angle, ${ }^{\circ} \ddagger$ & $31.5 \pm 14.4$ & $26.9 \pm 9.2$ & 0.226 \\
\hline Correction of kyphosis, $^{\circ}$ & $17.5 \pm 18.4$ & $16.0 \pm 17.1$ & 0.802 \\
\hline Length of op, mins & $233.5 \pm 49.6$ & $265.0 \pm 70.3$ & 0.360 \\
\hline Estimated blood loss, ml & $1026 \pm 706$ & $1228 \pm 965$ & 0.524 \\
\hline Length of stay in the hospital, days & $16.8 \pm 6.1$ & $18.5 \pm 4.5$ & 0.318 \\
\hline Total hospital charges, $\$$ & $14,581.60 \pm 1821.80$ & $16,310.30 \pm 1462.40$ & 0.002 \\
\hline Implant charges, \$ & $9194.50 \pm 1018.20$ & $11,040.60 \pm 1191.30$ & 0.000 \\
\hline
\end{tabular}

Values are expressed as the mean \pm SD.

* Statistically significant at $\alpha=0.05$ level.

$\dagger$ Statistically significant at $\alpha=0.01$ level.

‡ T5-12 kyphosis. 

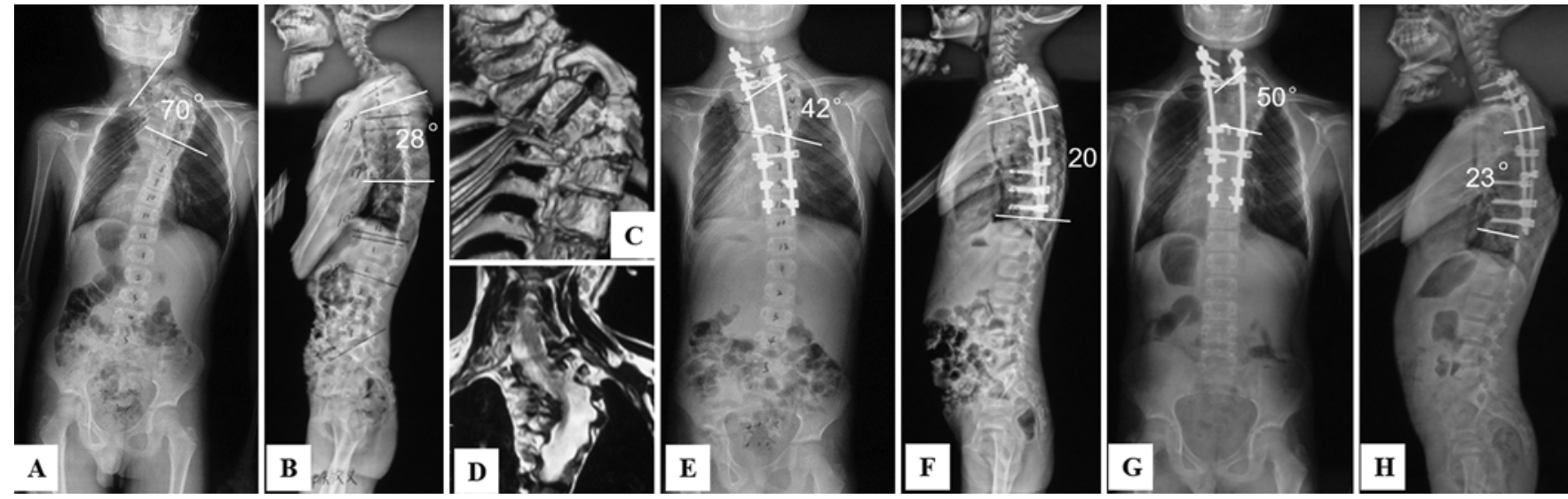

FIG. 2. A 10 -year-old boy with NF1 was surgically treated with a 1.00 implant density (LD group). The preoperative thoracic Cobb angle was $70^{\circ}(\mathbf{A})$, with a $28^{\circ}$ kyphotic curve (B). Radiographs (A and B), CT scan (C), and MR image (D) indicated dystrophic changes, including rib penciling, vertebral scalloping and wedging, and so on. Postoperative radiographs (E and F) showed a $40 \%$ primary curve correction rate (from $70^{\circ}$ preoperatively to $42^{\circ}$ postoperatively). A coronal correction loss of $8^{\circ}$ was observed after 2 years of follow-up ( $\mathbf{G}$ and $\mathbf{H})$.

Recently, Suk et al. reported fewer screw pullouts and pedicle fractures during rod de-rotation maneuvers with high implant density on the concave side, which resulted in an additional $10 \%$ curve correction for the idiopathic thoracic curve. ${ }^{20}$ To date, most of the findings regarding the correlation between implant density and curve correction have been based on the idiopathic scoliosis population. No study, to our knowledge, has specifically focused on the correlation between implant density and curve correction in patients with dystrophic NF1-associated scoliosis.

The insertion of pedicle screws can be difficult in patients with dystrophic NF1-associated scoliosis because landmarks and anatomies of the vertebrae and pedicle are seriously distorted, with narrow and inconsistent pedicle shapes. ${ }^{15}$ Moreover, decreased bone mineral density in patients with NF1-associated scoliosis makes it difficult to anchor the implants securely. ${ }^{11}$ Given the innate weakness and softness of the vertebral bone structure, loss of the surgical correction seems to be a common phenomenon during the postoperative course, particularly in those with low implant density. ${ }^{10} \mathrm{Hsu}$ et al. reported a $15 \%$ correction loss in the apical region in patients with dystrophic NF1 with low implant density. ${ }^{10}$

Image-guided spinal navigation systems provide highquality intraoperative 3D images, which has significantly increased the accuracy and safety of pedicle screw insertion..$^{12}$ In our scoliosis center, the $\mathrm{O}$-arm navigation system has been used since December 2012 for the insertion of difficult pedicle screws in patients with severe kyphoscoliosis, congenital scoliosis, NF1, and others. With the help of the O-arm, higher implant density could be achieved more safely. In this study, the $\mathrm{O}$-arm navigation system
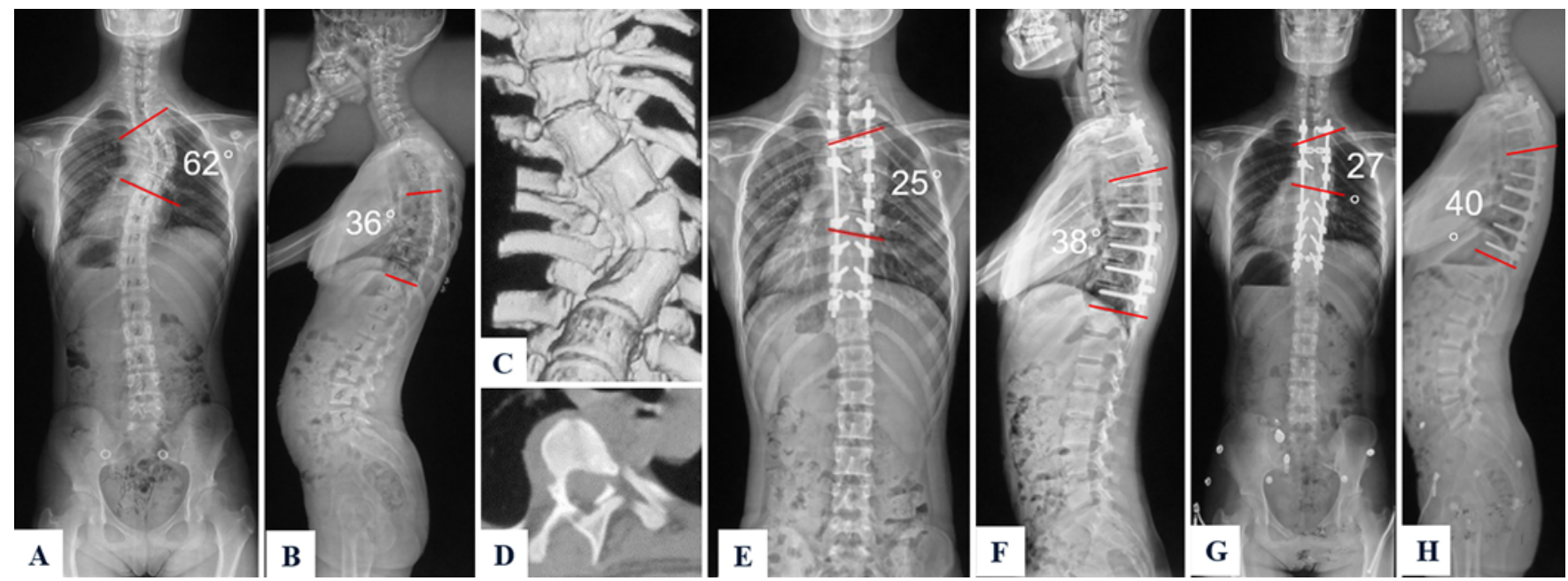

FIG. 3. A 13-year-old girl with NF1 was surgically treated with a 1.66 implant density (HD group). The preoperative thoracic Cobb angle was $62^{\circ}(\mathbf{A})$, with a $36^{\circ}$ kyphotic curve (B). CT scans indicated dystrophic changes, including rib penciling, vertebral scalloping and wedging, spindling of the transverse processes, and so on ( $C$ and $\mathbf{D})$. Postoperative radiographs ( $E$ and $F$ ) showed a $59.7 \%$ primary curve correction rate (from $62^{\circ}$ preoperatively to $25^{\circ}$ postoperatively). A coronal correction loss of $2^{\circ}$ was observed after 2.1 years of follow-up ( $\mathbf{G}$ and $\mathbf{H}$ ). Figure is available in color online only. 
TABLE 4. Comparison of preoperative and postoperative 2-year follow-up SRS-22 scores between LD and HD groups

\begin{tabular}{|c|c|c|c|c|c|c|c|}
\hline \multirow{2}{*}{$\begin{array}{l}\text { SRS-22 } \\
\text { Domain }\end{array}$} & \multicolumn{3}{|c|}{ LD Group } & \multicolumn{3}{|c|}{ HD Group } & \multirow{2}{*}{$\begin{array}{c}p \\
\text { Valuef }\end{array}$} \\
\hline & Preop Score & Postop Score & p Value* & Preop Score & Postop Score & p Value $\dagger$ & \\
\hline Appearance & $3.3 \pm 0.19(2.9-3.5)$ & $4.1 \pm 0.14(3.9-4.4)$ & $0.000 \S$ & $3.3 \pm 0.21(2.9-3.5)$ & $4.3 \pm 0.18(3.9-4.5)$ & $0.000 \S$ & 0.055 \\
\hline Activity & $4.1 \pm 0.19(3.9-4.3)$ & $4.3 \pm 0.21(4.0-4.5)$ & $0.003 \S$ & $4.1 \pm 0.16(3.9-4.2)$ & $4.4 \pm 0.18(4.1-4.6)$ & $0.000 \S$ & 0.109 \\
\hline Pain & $4.1 \pm 0.23(3.9-4.3)$ & $4.1 \pm 0.18(3.9-4.3)$ & 0.968 & $4.2 \pm 0.19(3.9-4.3)$ & $4.2 \pm 0.17(3.9-4.3)$ & 0.998 & 0.120 \\
\hline Mental health & $3.8 \pm 0.16(3.4-4.2)$ & $4.2 \pm 0.17(3.9-4.5)$ & $0.000 \S$ & $3.7 \pm 0.18(3.4-4.3)$ & $4.3 \pm 0.21(3.9-4.6)$ & $0.000 \S$ & 0.103 \\
\hline Satisfaction & - & $4.2 \pm 0.18(3.9-4.4)$ & - & - & $4.3 \pm 0.15(3.9-4.5)$ & - & 0.060 \\
\hline Total score & - & $4.2 \pm 0.17(4.0-4.4)$ & - & - & $4.3 \pm 0.18(4.2-4.5)$ & - & 0.075 \\
\hline
\end{tabular}

- = not available

Data are expressed as the mean \pm SD (range).

* Compared with preoperative score and postoperative score in LD group.

$\dagger$ Compared with preoperative score and postoperative score in HD group.

$\ddagger$ Compared with postoperative score of LD group and postoperative score of HD group.

$\S$ Reached statistical significance.

was used in 17 patients, with a mean implant density of $1.58 \pm 0.09$, which was higher than the average level.

For the first time, to our knowledge, our study investigated if higher implant density leads to better curve correction and clinical outcomes in patients with dystrophic scoliosis secondary to NF1. Results in this retrospective cohort showed that implant density was significantly correlated with the immediate postoperative coronal correction and loss of correction at final follow-up. Compared with the LD group, patients in the HD group had better coronal correction rates $(45.2 \% \pm 14.7 \%$ vs $55.2 \% \pm 16.4 \%$; $\mathrm{p}<0.05)$ and less correction loss $\left(6.5^{\circ} \pm 2.6^{\circ}\right.$ vs $4.1^{\circ} \pm 2.8^{\circ}$; $\mathrm{p}<0.05)$.

Conceivably, more anchor points for internal fixation can sustain more internal stress, and may have contributed to the superior coronal correction and maintenance at follow-up in patients with higher implant density. In addition, our study found that the SRS-22 scores were significantly improved in both groups at final follow-up with respect to the appearance, activity, and mental health domains. However, no statistical difference was found between the groups in total score or in any of the 5 domains at final follow-up.

A possible reason for this phenomenon is that both groups achieved a satisfactory curve correction from the viewpoint of the patients; thus the potential difference can be covered in this limited patient cohort, even though the HD group showed a better outcome on the radiographs. Besides, although many studies have proven the reliability and validity of the SRS-22 questionnaire, ${ }^{18,21}$ the limitation of its sensitivity still exists, which cannot distinguish minuscule differences.

A power analysis was conducted by Carreon et al., which took into account the minimal clinically important difference (MCID) of the SRS-22 outcome instrument. ${ }^{5}$ In this study, both groups achieved the MCID in the activity domain (0.2 in the LD group and 0.3 in the HD group), which was defined as 0.08 . Moreover, the HD group also achieved the MCID in the appearance domain, which was defined as 0.98 . In addition, the difference in the appearance domain between the HD and LD groups at last followup was close to the marginal significant difference. From this point, we can infer that the HD group may achieve more improvement in the appearance domain. However, it is expected to be confirmed by a large patient cohort and longer follow-up.

A disadvantage of higher implant density is the increased cost. Although the higher-density implants were more expensive, better coronal correction and less correction loss were observed at the last follow-up. On the other hand, the mean follow-up of the cohort was only $28.8 \pm$ 4.3 months. We assumed that additional benefits, including low reoperation rates and better maintenance in coronal and sagittal balance, might be observed during longerterm follow-up.

Limitations of the present study include the retrospective nature of the review and the relatively short follow-up. Because the surgical management of spinal deformities in neurofibromatosis is a major challenge with a high incidence of pseudarthrosis and curve progression, ${ }^{1,4}$ loss of correction and curve progression were reported even in the presence of solid fusion..$^{10}$ Thus, whether implant density affects radiographic and clinical outcomes in patients with dystrophic scoliosis secondary to NF1 merits further investigation with longer follow-up. In addition, 7 patients $(17 \%)$ were treated with screws and hooks, which may have influenced the correction rate.

\section{Conclusions}

Although the total SRS-22 score was improved in both groups at final follow-up, no significant difference between the HD and LD groups was found in any of the SRS-22 domains at last follow-up. However, the present study demonstrated that higher implant density was correlated with superior coronal correction and less postoperative correction loss in patients with dystrophic NF1-associated scoliosis. There seemed to be better radiographic outcomes with the higher implant-density constructs despite increased hospital charges.

\section{Acknowledgments}

This work was supported by the National Natural Science Foundation of China (grant no. 81371912) and the Natural Science Foundation of Jiangsu Province (grant no. BK20160122). 


\section{References}

1. Betz RR, Iorio R, Lombardi AV, Clancy M, Steel HH: Scoliosis surgery in neurofibromatosis. Clin Orthop Relat Res (245):53-56, 1989

2. Bharucha NJ, Lonner BS, Auerbach JD, Kean KE, Trobisch PD: Low-density versus high-density thoracic pedicle screw constructs in adolescent idiopathic scoliosis: do more screws lead to a better outcome? Spine J 13:375-381, 2013

3. Boyd KP, Korf BR, Theos A: Neurofibromatosis type 1. J Am Acad Dermatol 61:1-14, quiz 15-16, 2009

4. Calvert PT, Edgar MA, Webb PJ: Scoliosis in neurofibromatosis. The natural history with and without operation. J Bone Joint Surg Br 71:246-251, 1989

5. Carreon LY, Sanders JO, Diab M, Sucato DJ, Sturm PF, Glassman SD: The minimum clinically important difference in Scoliosis Research Society-22 Appearance, Activity, And Pain domains after surgical correction of adolescent idiopathic scoliosis. Spine (Phila Pa 1976) 35:2079-2083, 2010

6. Clements DH, Betz RR, Newton PO, Rohmiller M, Marks $\mathrm{MC}$, Bastrom T: Correlation of scoliosis curve correction with the number and type of fixation anchors. Spine (Phila Pa 1976) 34:2147-2150, 2009

7. Funasaki H, Winter RB, Lonstein JB, Denis F: Pathophysiology of spinal deformities in neurofibromatosis. An analysis of seventy-one patients who had curves associated with dystrophic changes. J Bone Joint Surg Am 76:692-700, 1994

8. Gajeski BL, Kettner NW, Awwad EE, Boesch RJ: Neurofibromatosis type I: clinical and imaging features of Von Recklinghausen's disease. J Manipulative Physiol Ther 26:116-127, 2003

9. Gebhart S, Alton TB, Bompadre V, Krengel WF: Do anchor density or pedicle screw density correlate with short-term outcome measures in adolescent idiopathic scoliosis surgery? Spine (Phila Pa 1976) 39:E104-E110, 2014

10. Hsu LC, Lee PC, Leong JC: Dystrophic spinal deformities in neurofibromatosis. Treatment by anterior and posterior fusion. J Bone Joint Surg Br 66:495-499, 1984

11. Illés T, Halmai V, de Jonge T, Dubousset J: Decreased bone mineral density in neurofibromatosis-1 patients with spinal deformities. Osteoporos Int 12:823-827, 2001

12. Jin M, Liu Z, Liu X, Yan H, Han X, Qiu Y, et al: Does intraoperative navigation improve the accuracy of pedicle screw placement in the apical region of dystrophic scoliosis secondary to neurofibromatosis type I: comparison between O-arm navigation and free-hand technique. Eur Spine J 25:17291737,2016

13. Kuorilehto T, Pöyhönen M, Bloigu R, Heikkinen J, Väänänen $\mathrm{K}$, Peltonen J: Decreased bone mineral density and content in neurofibromatosis type 1: lowest local values are located in the load-carrying parts of the body. Osteoporos Int 16:928936, 2005

14. Lammert M, Kappler M, Mautner VF, Lammert K, Störkel $\mathrm{S}$, Friedman JM, et al: Decreased bone mineral density in patients with neurofibromatosis 1. Osteoporos Int 16:11611166,2005

15. Li M, Fang X, Li Y, Ni J, Gu S, Zhu X: Successful use of posterior instrumented spinal fusion alone for scoliosis in 19 patients with neurofibromatosis type-1 followed up for at least 25 months. Arch Orthop Trauma Surg 129:915-921, 2009

16. Lykissas MG, Schorry EK, Crawford AH, Gaines S, Rieley $\mathrm{M}$, Jain VV: Does the presence of dystrophic features in patients with type 1 neurofibromatosis and spinal deformities increase the risk of surgery? Spine (Phila Pa 1976) 38:15951601,2013

17. Nash CL Jr, Moe JH: A study of vertebral rotation. J Bone Joint Surg Am 51:223-229, 1969

18. Qiu G, Qiu Y, Zhu Z, Liu Z, Song Y, Hai Y, et al: Re-evaluation of reliability and validity of simplified Chinese version of SRS-22 patient questionnaire: a multicenter study of 333 cases. Spine (Phila Pa 1976) 36:E545-E550, 2011

19. Quan GM, Gibson MJ: Correction of main thoracic adolescent idiopathic scoliosis using pedicle screw instrumentation: does higher implant density improve correction? Spine (Phila Pa 1976) 35:562-567, 2010

20. Suk SI, Lee SM, Chung ER, Kim JH, Kim SS: Selective thoracic fusion with segmental pedicle screw fixation in the treatment of thoracic idiopathic scoliosis: more than 5-year follow-up. Spine (Phila Pa 1976) 30:1602-1609, 2005

21. Zhao L, Zhang Y, Sun X, Du Q, Shang L: The Scoliosis Research Society-22 questionnaire adapted for adolescent idiopathic scoliosis patients in China: reliability and validity analysis. J Child Orthop 1:351-355, 2007

\section{Disclosures}

The authors report no conflict of interest concerning the materials or methods used in this study or the findings specified in this paper.

\section{Author Contributions}

Conception and design: Z Zhu, Li, Qiu. Acquisition of data: Li, Yuan, Sha, W Zhu. Analysis and interpretation of data: Li, Yuan, Sha, W Zhu. Drafting the article: Li, Sha. Critically revising the article: Z Zhu, Li. Reviewed submitted version of manuscript: Z $\mathrm{Zhu}, \mathrm{Li}$. Approved the final version of the manuscript on behalf of all authors: Z Zhu. Statistical analysis: Li, W Zhu. Administrative/technical/material support: Li. Study supervision: Z Zhu, Sha, Liu, Qiu, Wang, Yu.

\section{Correspondence}

Zezhang Zhu, Spine Surgery, Drum Tower Hospital, Nanjing University Medical School, 321 Zhongshan Rd., Nanjing 210008, China. email: zhuzezhang@126.com. 